\title{
An intriguing mass associated with a cleft palate.
}

\author{
Malek Daldoul ${ }^{1}$, Sirine $\operatorname{Tritar}^{1}$, Samia Ayachi ${ }^{1}$, Ramzi Moatemri $^{1}$, and Habib Khochteli ${ }^{1}$ \\ ${ }^{1}$ Sahloul University Hospital
}

March 3, 2022

\begin{abstract}
Nasopharyngeal teratomas (NPT) are rare and represent only $2 \%$ of all teratomas. They can lead to an embryopathogenic mechanical obstacle responsible for a cleft palate $(\mathrm{CP})$. We report the case of a 14-month-old female with NPT associated with a CP.
\end{abstract}

Teratoma is a tumor representing differentiation from all three embryonic germ layers, ectoderm, mesoderm, and endoderm ${ }^{1}$. Nasopharyngeal teratoma (NPT) can extend to the sphenoidal, endobuccal and pharyngeal regions. The most common associated abnormality is cleft palate $(\mathrm{CP})^{2}$.

A 14-month-old newborn girl was referred to out department for a large CP. She was born by caesarean delivery for previous uterine scar at 40 weeks of amenorrhea with birth weight $3 \mathrm{~kg}$. Oral examination revealed a firm mass of $1 \mathrm{x} 1.5 \mathrm{~cm}$, appended to the ethmoid (Figure 1.A). A head computed tomography scan revealed a well-delimited and heterogeneous tumor with calcifications and fat tissue, expanding to the sphenoid (Figure 1.C, 1.D).

Surgical excision of the tumor was performed immediately (Figure 1.B). The hard and soft palates were cleft with the mass occupying the space between the palatal shelves. Histologically the tumor was a true teratoma according to the Wittstock et al classification ${ }^{2}$.

The reconstruction of the velar and the palatal defect were performed 6 months later to ensure a complete excision. No recurrence of the mass has been observed during two years of follow-up.

NPT is rare and CP is the most common associated abnormality. The factors that may promote this association remain uncertain but the hypothesis of mechanical origin is the most plausible.

NPT could be an obstacle for the fusion of the palatal shelves during the development of the mammalian palate. It is also possible that the tumor may coexist with $\mathrm{CP}$.

\section{References :}

1. He J, Wang Y, Zhu H, Qiu W, He Y. Nasopharyngeal teratomas associated with cleft palate in newborn: report of 2 cases. Oral Surg Oral Med Oral Pathol Oral Radiol Endod. 2010;109:211-6.

2. C Diakité, H Bénateau, S Dakpé, P Guerreschi, P Galinier, A Veyssière. Management of nasopharyngeal teratomas associated with clefts palate. Int J Oral Maxillofac Surg 2019;48:291-297. 


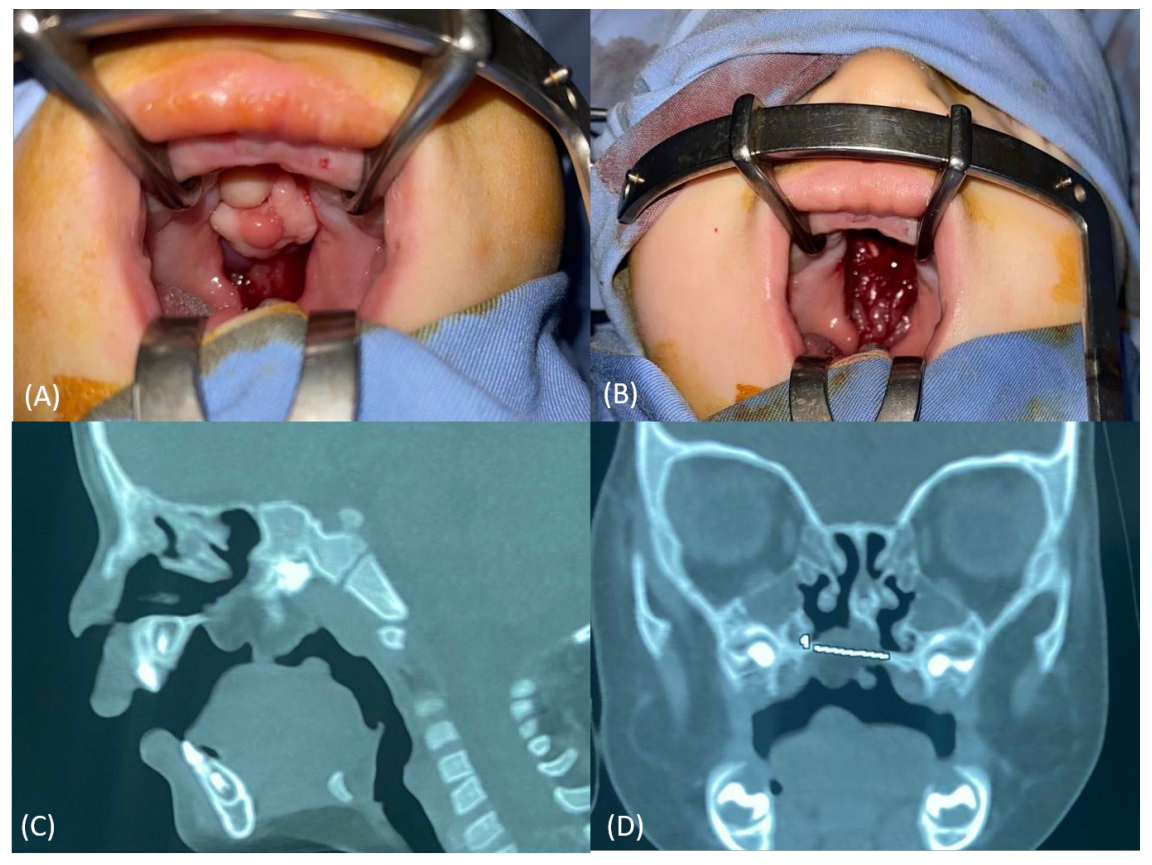

